(C)2009 IEEE. Personal use of this material is permitted. However, permission to reprint/republish this material for advertising or promotional purposes or for creating new collective works for resale or redistribution to servers or lists, or to reuse any copyrighted component of this work in other works must be obtained from the IEEE. 


\title{
On the Realization of 2-D Linear Systems With Recursively Computable Latent Variable Models
}

\author{
Ran Yang, Member, IEEE, Lorenzo Ntogramatzidis, and Michael Cantoni, Member, IEEE
}

\begin{abstract}
In this paper, a new latent variable model is proposed for the realization of 2-D single-input-single-output linear causal systems. For the general $(n, m)$ th-order 2-D system, the latent variable model obtained here is implicit when $n \neq m$. Importantly, however, even in this case, the model is recursively computable. The advantage of the proposed realization is that the dimension of the latent variable is $n m$, which is much smaller than that for existing latent variable realizations, such as Fornasini-Marchesini (FM) and Roesser models. Given a particular 2-D system, an algorithm is developed for further reducing the size of the realization.
\end{abstract}

Index Terms-Fornasini-Marchesini (FM) models, recursive latent variable models, 2 -D realizations.

\section{INTRODUCTION}

$\mathbf{I}$ N THE PAST two decades, 2-D latent variable models have been widely used in the analysis and synthesis of 2-D linear systems, including stability analysis [4], [26], digital filter design [13], [21], stabilization [6], [32], estimation [6], optimal control [6], [25], and the modelling of partial differential equations [5], [22], [28], [32].

A motivation for the use of latent variable representations of causal input-output processes-within both a 1- and a 2-D context-lies in the possibility of computing the output of a system recursively, given the input. Moreover, the recursive structure of latent variable models is often exploited when dealing with optimal synthesis problems (e.g., via dynamic programming). As such, one fundamental issue of systems and control theory is the realization of input-output transfer function representations in terms of latent variable models. While in the 1-D case the latent variable model that is usually employed is the classic state-space model, in the 2-D case, there are more degrees of

Manuscript received November 06, 2007; revised April 08, 2008. First published August 04, 2008; current version published March 11, 2009. This work was supported in part by the Australian Research Council under Grant DP0664789 and Grant DP0986577, by the National Natural Science Foundation of China under Grant 60504022, and by the Natural Science Foundation of Guangdong Province under Grant 05003343 . This paper was recommended by Associate Editor A. Kummert.

R. Yang is with the School of Information Science and Technology, Sun Yat-Sen University, Guangzhou 510275, China (e-mail: yangran@mail.sysu. edu.cn).

L. Ntogramatzidis is with the Department of Mathematics and Statistics, Curtin University of Technology, Perth, WA 6845, Australia (e-mail: 1.ntogramatzidis@curtin.edu.au).

M. Cantoni is with the Department of Electrical and Electronic Engineering, The University of Melbourne, Parkville, Vic. 3010, Australia (e-mail: cantoni@unimelb.edu.au).

Digital Object Identifier 10.1109/TCSI.2008.2002546 freedom in the choice of the type of the latent variable model to be employed. In fact, the models that are commonly utilized for the realization of causal 2-D transfer functions are the Roesser model [31], the Fornasini-Marchesini first (FM-I) model [7] and the second (FM-II) model [8], and the Fornasini-Marchesini model in Kurek form [20] (see also [1], [12], [17], [18], and [34]). These models are not fully independent, as each can be embedded in the other, as shown, for example, in [10]. Both Roesser and Fornasini-Marchesini models exhibit a recursive structure, given appropriate (single-sided) boundary conditions.

In this paper, we show that causal 2-D single-input-singleoutput (SISO) transfer functions can be realized in terms of a new implicit latent variable model which retains a recursive structure for the computation of the input-output relation, given appropriate boundary conditions. Note that, in general, 2-D implicit models do not enjoy this property. In other words, for implicit latent variable models, a recursive technique for the solution of the associated one or 2-D difference equation may not exist (see [11], [15], [23], [24], [27], [29], and the references therein for the 1-D and the 2-D case, respectively). In fact, implicit models are usually employed when the process to be realized displays a noncausal relation between its inputs and outputs, or to capture impulsive behavior. The relation between the concepts of causality and recursibility in the 2-D case is investigated in detail in [24].

For the realization problem considered in this paper, by allowing the latent variable model to take an implicit form, we obtain a recursively computable realization with size smaller than those of the standard realizations given in terms of Roesser or Fornasini-Marchesini models. More precisely, for a causal 2-D system described by an $(n, m)$ th-order transfer function, a realization with latent variable dimension $n m$ is provided. It is also shown that the size of this realization may be further reduced if some coefficients appearing in the transfer function are zero. Furthermore, for this purpose, an algorithm is developed for the reduction of the size of the realization in these particular cases.

The motivation for developing the realization technique presented here with implicit models is illustrated in the following by an example.

Example 1: Let $\mathbb{N}$ be the set of nonnegative integer numbers. Consider the 2-D sequences $\left(u_{i, j}\right)_{i, j \in \mathbb{N}}$ and $\left(y_{i, j}\right)_{i, j \in \mathbb{N}}$ which are representative of the input and the output of the $(1,3)$ th-order 2-D process ruled by

$$
\begin{gathered}
\left(\sum_{i=0}^{1} \sum_{j=0}^{3} a_{i, j} \lambda_{h}^{i} \lambda_{v}^{j}\right) Y\left(\lambda_{h}, \lambda_{v}\right)=\left(\sum_{i=0}^{1} \sum_{j=0}^{3} b_{i, j} \lambda_{h}^{i} \lambda_{v}^{j}\right) U\left(\lambda_{h}, \lambda_{v}\right) \\
a_{0,0}=1, \quad b_{0,0}=0
\end{gathered}
$$


TABLE I

COMPARISON OF THE SIZES OF DIFFERENT REALIZATIONS FOR (1)

\begin{tabular}{ccccc}
\hline model type & Roesser & FM-I & FM-II & FM-II \\
\hline realisation given in & {$[14]$} & {$[7]$} & {$[3]$} & {$[33]$} \\
\hline size of realisation & 6 & 16 & 14 & 7 \\
\hline
\end{tabular}

where $U\left(\lambda_{h}, \lambda_{v}\right)$ and $Y\left(\lambda_{h}, \lambda_{v}\right)$ are formal power series associated with the two sequences $u_{i, j}$ and $y_{i, j}$, respectively, by means of the transformation

$$
S\left(\lambda_{h}, \lambda_{v}\right):=\sum_{i=0}^{\infty} \sum_{j=0}^{\infty} s_{i, j} \lambda_{h}^{i} \lambda_{v}^{j}
$$

that maps the bivariate sequence $\left(s_{i, j}\right)_{i, j \in \mathbb{N}}$ into the formal power series $S\left(\lambda_{h}, \lambda_{v}\right)$, and the coefficients $a_{i, j}$ and $b_{i, j}$ are real. Notice that with this definition of formal power series associated with 2-D sequences, the symbols $\lambda_{h}$ and $\lambda_{v}$ may be viewed as shift operators in the horizontal and the vertical direction, respectively. The sizes of the realizations (i.e., the dimension of the latent variable vector) obtained for different model structures and via different techniques are shown in Table I.

Now, consider the latent variable model

$$
\begin{aligned}
& {\left[\begin{array}{ll|l}
0 & 0 & 0 \\
0 & 0 & 0 \\
\hline 0 & 0 & 1
\end{array}\right]\left[\frac{x_{i+1, j+1}^{\prime}}{x_{i+1, j+1}^{\prime \prime}}\right] } \\
&= {\left[\begin{array}{ll|l}
0 & 0 & -a_{1,3} \\
1 & 0 & -a_{1,2} \\
\hline 0 & 1 & -a_{1,1}
\end{array}\right]\left[\frac{x_{i, j}^{\prime}}{x_{i, j}^{\prime \prime}}\right]+\left[\begin{array}{ll|l}
0 & 0 & -a_{0,3} \\
0 & 0 & -a_{0,2} \\
\hline 0 & 0 & -a_{0,1}
\end{array}\right]\left[\frac{x_{i+1, j}^{\prime}}{x_{i+1, j}^{\prime \prime}}\right] } \\
&+\left[\begin{array}{rr|r}
-1 & 0 & 0 \\
0 & -1 & 0 \\
\hline 0 & 0 & -a_{1,0}
\end{array}\right]\left[\frac{x_{i, j+1}^{\prime}}{x_{i, j+1}^{\prime \prime}}\right]+\left[\begin{array}{l}
b_{1,3} \\
\frac{b_{1,2}}{b_{1,1}}
\end{array}\right] u_{i, j} \\
&+\left[\begin{array}{c}
b_{0,3} \\
\frac{b_{0,2}}{b_{0,1}}
\end{array}\right] u_{i+1, j}+\left[\frac{0}{b_{1,0}}\right] u_{i, j+1} \\
& y_{i, j}=\left[\begin{array}{ll|l}
0 & 0 & 1
\end{array}\right]\left[\frac{x_{i, j}^{\prime}}{x_{i, j}^{\prime \prime}}\right]
\end{aligned}
$$

with the boundary conditions $x_{i, j}^{\prime}=0, x_{i, j}^{\prime \prime}=0$, and $u_{i, j}=0$ for all $(i, j) \in[-1, \infty) \times\{-1\} \cup\{-1\} \times[-1, \infty)$. Notice that this model is implicit. However, with the boundary conditions specified, it is still causal in terms of the input-output relationship, since the first equation can be written explicitly, as seen in

$$
\begin{aligned}
z_{i, j}= & -\sum_{k=0}^{1} \sum_{l=2}^{3} a_{k, l} x_{i+1-k, j+1-l}^{\prime \prime} \\
& +\sum_{k=0}^{1} \sum_{l=2}^{3} b_{k, l} u_{i+1-k, j+1-l}
\end{aligned}
$$

and (4), shown at the bottom of the page, where $z_{i, j}:=$ $\left[\begin{array}{ll}0 & 1\end{array}\right] x_{i, j}^{\prime}$. By substitution of (3) into (4), and by taking the boundary conditions into account, the input-output relation of the model thus obtained is found to be consistent with (1). In other words, the latent variable model obtained is a realization of (1), and its size is 3 , which is much smaller than the orders of the realizations shown in Table I.

Observe that if $z_{i, j}$ is removed from (4), together with the output equation, (4) represents a $(1,1)$ st-order 2 -D system, i.e.,

$$
\begin{array}{r}
\left(1+a_{1,0} \lambda_{h}+a_{0,1} \lambda_{v}+a_{1,1} \lambda_{h} \lambda_{v}\right) Y\left(\lambda_{h}, \lambda_{v}\right) \\
=\left(b_{1,0} \lambda_{h}+b_{0,1} \lambda_{v}+b_{1,1} \lambda_{h} \lambda_{v}\right) U\left(\lambda_{h}, \lambda_{v}\right) .
\end{array}
$$

The addition of another latent variable $z_{i, j}$ yields a realization for the $(1,3)$ th-order system (1). Following this idea, a new realization method can be developed for the general $(n, m)$ th-order 2-D system

$$
p\left(\lambda_{h}, \lambda_{v}\right) Y\left(\lambda_{h}, \lambda_{v}\right)=q\left(\lambda_{h}, \lambda_{v}\right) U\left(\lambda_{h}, \lambda_{v}\right)
$$

where the polynomials $p\left(\lambda_{h}, \lambda_{v}\right)$ and $q\left(\lambda_{h}, \lambda_{v}\right)$ are defined by

$$
\begin{aligned}
p\left(\lambda_{h}, \lambda_{v}\right) & :=\sum_{i=0}^{n} \sum_{j=0}^{m} a_{i, j} \lambda_{h}^{i} \lambda_{v}^{j}, \quad a_{0,0}=1 \\
q\left(\lambda_{h}, \lambda_{v}\right) & :=\sum_{i=0}^{n} \sum_{j=0}^{m} b_{i, j} \lambda_{h}^{i} \lambda_{v}^{j}
\end{aligned}
$$

and where $n$ is not necessarily equal to $m$. The latent variable model used here is of the implicit form

$$
\begin{aligned}
E x_{i+1, j+1}= & A_{0} x_{i, j}+A_{1} x_{i+1, j}+A_{2} x_{i, j+1}+B_{0} u_{i, j} \\
& +B_{1} u_{i+1, j}+B_{2} u_{i, j+1} \\
y_{i, j}= & C x_{i, j}+D u_{i, j}
\end{aligned}
$$

with the following boundary conditions:

$$
x_{i, j}=0 \quad u_{i, j}=0 \quad \forall(i, j) \in \mathbb{B}
$$

$$
x_{i+1, j+1}^{\prime \prime}=z_{i, j}+\sum_{(k, l) \in\{(0,1),(1,0),(1,1)\}}\left(-a_{k, l} x_{i+1-k, j+1-l}^{\prime \prime}+b_{k, l} u_{i+1-k, j+1-l}\right)
$$


where $\mathbb{B}:=[-1, \infty) \times\{-1\} \cup\{-1\} \times[-1, \infty)$. In Section III, it is shown that the solution of (6) with boundary conditions (7) is uniquely determined and can be computed recursively.

When $n=m, E$ is an identity matrix. When $n<m$, the matrices of the model (6) have the following structure:

$$
\begin{array}{rlrl}
E & =\left[\begin{array}{ll}
0 & 0 \\
0 & I
\end{array}\right] & A_{0}=\left[\begin{array}{cc}
A_{0}^{22} & A_{0}^{21} \\
A_{0}^{12} & A_{0}^{11}
\end{array}\right] \\
A_{1}=\left[\begin{array}{ll}
0 & A_{1}^{21} \\
0 & A_{1}^{11}
\end{array}\right] \quad A_{2}=\left[\begin{array}{cc}
-I & 0 \\
0 & A_{2}^{11}
\end{array}\right] \\
B_{0}=\left[\begin{array}{l}
B_{0}^{2} \\
B_{0}^{1}
\end{array}\right] \quad B_{1}=\left[\begin{array}{l}
B_{1}^{2} \\
B_{1}^{1}
\end{array}\right] \quad B_{2}=\left[\begin{array}{c}
0 \\
B_{2}^{1}
\end{array}\right] .
\end{array}
$$

When $n>m$, the matrices of the latent variable model (6) have the following structure:

$$
\begin{aligned}
E & =\left[\begin{array}{ll}
0 & 0 \\
0 & I
\end{array}\right] \quad A_{0}=\left[\begin{array}{ll}
A_{0}^{22} & A_{0}^{21} \\
A_{0}^{12} & A_{0}^{11}
\end{array}\right] \\
A_{1} & =\left[\begin{array}{cc}
-I & 0 \\
0 & A_{1}^{11}
\end{array}\right] \quad A_{2}=\left[\begin{array}{ll}
0 & A_{2}^{21} \\
0 & A_{2}^{11}
\end{array}\right] \\
B_{0} & =\left[\begin{array}{c}
B_{0}^{2} \\
B_{0}^{1}
\end{array}\right] \quad B_{1}=\left[\begin{array}{c}
0 \\
B_{1}^{1}
\end{array}\right] \quad B_{2}=\left[\begin{array}{l}
B_{1}^{2} \\
B_{2}^{1}
\end{array}\right] .
\end{aligned}
$$

The details of a realization for the input-output model (5) in the latent variable model (6) with boundary conditions (7) are given in Section II. Based on this, an algorithm is developed to reduce the size of the realization for 2-D polynomial input-output models with particular zero structure, as described in Section IV.

\section{MAIN RESUltS}

A solution to the realization problem for the 2-D input-output model (5) in terms of the general latent variable model (6) is given in the next theorem.

Theorem 2.1: Given an $(n, m)$ th-order 2-D system in the form of (5), a realization in terms of the model (6) with boundary conditions (7) of size $n \cdot m$ is given by the following matrices:

$$
\begin{aligned}
A_{k} & =\left[\begin{array}{ccccc}
A_{k}{ }^{(r, r)} & \mathbf{0} & \ldots & \mathbf{0} & A_{k}^{(r, 1)} \\
A_{k}^{(r-1, r)} & \ddots & \ddots & \vdots & \vdots \\
\mathbf{0} & \ddots & \ddots & \mathbf{0} & \vdots \\
\vdots & \ddots & A_{k}^{(2,3)} & A_{k}^{(2,2)} & A_{k}^{(2,1)} \\
\mathbf{0} & \cdots & \mathbf{0} & A_{k}^{(1,2)} & A_{k}^{(1,1)}
\end{array}\right] \\
B_{k} & =\left[\begin{array}{c}
B_{k}^{(r)} \\
B_{k}^{(r-1)} \\
\vdots \\
B_{k}^{(2)} \\
B_{k}^{(1)}
\end{array}\right]
\end{aligned}
$$

for $k=0,1,2$ and

$$
E=\operatorname{diag}\left\{E^{(r, r)}, \ldots, E^{(1,1)}\right\} \quad C=\left[\begin{array}{lllll}
0 & \cdots & 0 & 0 & 1
\end{array}\right]
$$

with $r=\max (n, m), s=\min (n, m)$, and

$$
\begin{aligned}
& E^{(i, i)}= \begin{cases}I_{(2 i-1) \times(2 i-1)}, & i=1, \ldots, s \\
\mathbf{0}_{s \times s}, & i=s+1, \ldots, r\end{cases}
\end{aligned}
$$

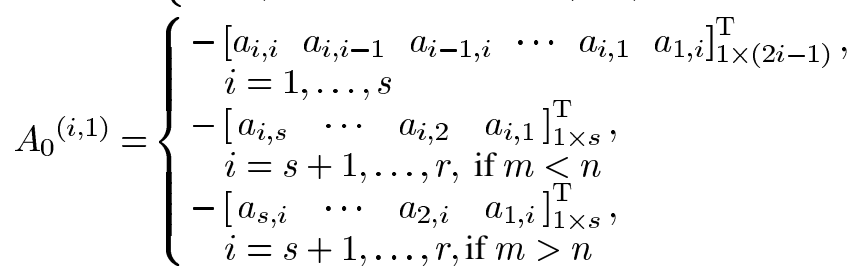

$$
\begin{aligned}
& A_{0}{ }^{(i-1, i)}=\left\{\begin{array}{c}
M_{(2 i-3) \times(2 i-1)}^{\prime}, \\
i=2, \ldots, s \\
M_{(2 s-1) \times s}^{\prime \prime}, \\
i=s+1, \text { if } n<m \\
M_{(2 s-1) \times s}^{\prime \prime \prime}, \\
i=s+1, \text { if } n>m \\
I_{s \times s}, \\
i=s+2, \ldots, r
\end{array}\right.
\end{aligned}
$$

with

$$
\begin{aligned}
& M_{(2 i-3) \times(2 i-1)}^{\prime}[k, l]= \begin{cases}1, & k=l=1 \\
0, & \text { otherwise }\end{cases} \\
& M_{(2 s-1) \times s}^{\prime \prime}[k, l]= \begin{cases}1, & k=l=1 \\
1, & k=2 l-1, l>1 \\
0, & \text { otherwise }\end{cases} \\
& M_{(2 s-1) \times s}^{\prime \prime \prime}[k, l]= \begin{cases}1, & k=l=1 \\
1, & k=2 l-2, l>1 \\
0, & \text { otherwise }\end{cases} \\
& A_{0}{ }^{(i, i)}= \begin{cases}\mathbf{0}_{(2 i-1) \times(2 i-1)}, & i=2, \ldots, s \\
\mathbf{0}_{s \times s}, & i=s+1, \ldots, r\end{cases}
\end{aligned}
$$

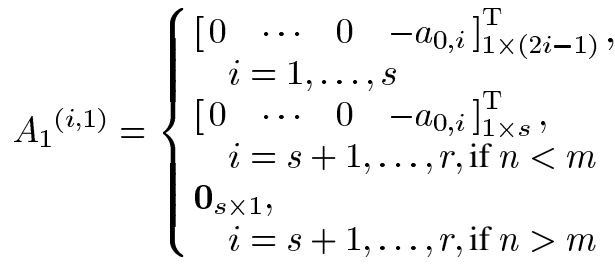

$$
\begin{aligned}
& A_{1}{ }^{(i-1, i)}=\left\{\begin{array}{c}
N_{(2 i-3) \times(2 i-1)}^{\prime}, \\
i=2, \ldots, s \\
\mathbf{0}_{(2 s-1) \times s}, \\
i=s+1 \\
\mathbf{0}_{s \times s}, \\
i=s+2, \ldots, r .
\end{array}\right.
\end{aligned}
$$


and

$$
\begin{aligned}
& N_{(2 i-3) \times(2 i-1)}^{\prime}[k, l]= \begin{cases}1, & l=k+2, k \text { is odd } \\
0, & \text { otherwise }\end{cases} \\
& A_{1}{ }^{(i, i)}=\left\{\begin{array}{c}
\mathbf{0}_{(2 i-1) \times(2 i-1)}, \\
i=2, \ldots, s \\
-I_{s \times s}, \\
i=s+1, \ldots, r, \text { if } n>m \\
\mathbf{0}_{s \times s}, \\
i=s+1, \ldots, r, \text { if } n<m
\end{array}\right.
\end{aligned}
$$

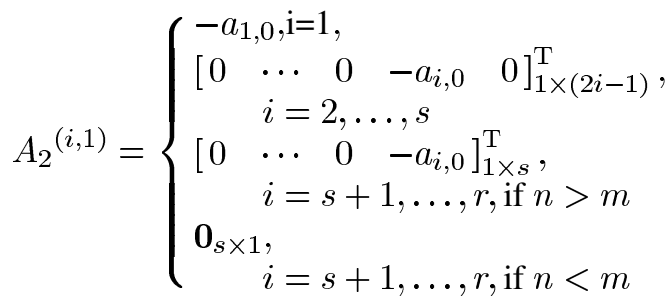

$$
\begin{aligned}
& A_{2}{ }^{(i-1, i)}=\left\{\begin{array}{c}
Z_{(2 i-3) \times(2 i-1)}, \\
i=2, \ldots, s \\
\mathbf{0}_{(2 s-1) \times s}, \\
i=s+1 \\
\mathbf{0}_{s \times s}, \\
i=s+2, \ldots, r
\end{array}\right.
\end{aligned}
$$

with

$$
\begin{aligned}
& Z_{(2 i-3) \times(2 i-1)}[k, l]=\left\{\begin{array}{l}
1, \quad k=1, l=2 \\
1, \quad l=k+2, k=2,4, \ldots, 2 i-4 \\
0, \quad
\end{array}\right. \\
& A_{2}{ }^{(i, i)}=\left\{\begin{array}{c}
\mathbf{0}_{(2 i-1) \times(2 i-1)}, \\
i=2, \ldots, s \\
-I_{s \times s}, \\
i=s+1, \ldots, r, \text { if } n<m \\
\mathbf{0}_{s \times s}, \\
i=s+1, \ldots, r, \text { if } n>m
\end{array}\right.
\end{aligned}
$$

and

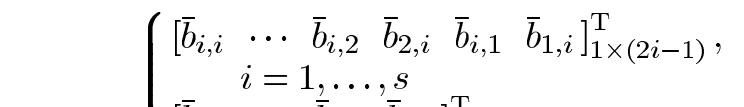

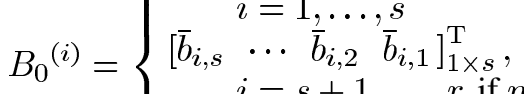

$$
\begin{aligned}
& i=s+1, \ldots, r \text {, if } n>m
\end{aligned}
$$$$
\left[\begin{array}{llll}
\bar{b}_{s, i} & \cdots & \bar{b}_{2, i} & \bar{b}_{1, i}
\end{array}\right]_{1 \times s}^{\mathrm{T}}
$$$$
i=s+1, \ldots, r, \text { if } n<m
$$

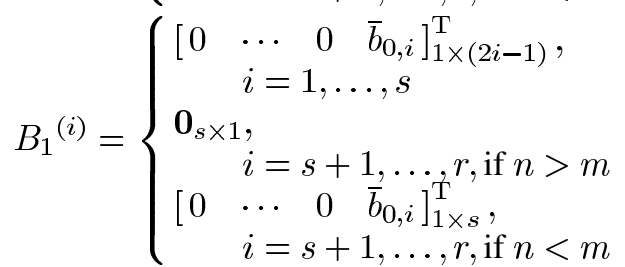

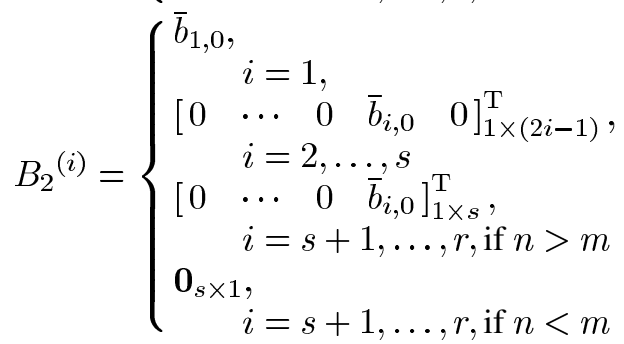$$
D=b_{0,0}
$$

where $\bar{b}_{i, j}:=b_{i, j}-a_{i, j} b_{0,0}$ for all $i=0,1, \ldots, n$ and $j=$ $0,1, \ldots, m$.
Proof: It can be shown by direct calculation that

$$
\begin{aligned}
& \operatorname{det}\left.H\left(\lambda_{h}, \lambda_{v}\right)\right) \\
&= \begin{cases}p\left(\lambda_{h}, \lambda_{v}\right), & n=m \\
\lambda_{h}^{n(m-n)} p\left(\lambda_{h}, \lambda_{v}\right), & n<m \\
\lambda_{v}^{m(n-m)} p\left(\lambda_{h}, \lambda_{v}\right), & n>m\end{cases} \\
& \text { Cadj } H\left(\lambda_{h}, \lambda_{v}\right) V\left(\lambda_{h}, \lambda_{v}\right) \\
& \text { where } \begin{array}{ll}
q\left(\lambda_{h}, \lambda_{v}\right)-D p\left(\lambda_{h}, \lambda_{v}\right), \\
\lambda_{h}^{n(m-n)}\left(q\left(\lambda_{h}, \lambda_{v}\right)-D p\left(\lambda_{h}, \lambda_{v}\right)\right), & n<m \\
\lambda_{v}^{m(n-m)}\left(q\left(\lambda_{h}, \lambda_{v}\right)-D p\left(\lambda_{h}, \lambda_{v}\right)\right), & n>m
\end{array} \\
& \\
& \quad H\left(\lambda_{h}, \lambda_{v}\right):=E-A_{0} \lambda_{h} \lambda_{v}-A_{1} \lambda_{v}-A_{2} \lambda_{h}
\end{aligned}
$$

(see [30] for the details). We now show that, in all three cases, (5) is satisfied. To this end, let $X\left(\lambda_{h}, \lambda_{v}\right), U\left(\lambda_{h}, \lambda_{v}\right)$, and $Y\left(\lambda_{h}, \lambda_{v}\right)$ denote the formal power series associated with the signals $x, u$, and $y$, respectively. Moreover, notice that by

$$
\sum_{i=0}^{\infty} \sum_{j=0}^{\infty} x_{i+1, j} \lambda_{h}^{i} \lambda_{v}^{j}=\sum_{k=1}^{\infty} \sum_{j=0}^{\infty} x_{k, j} \lambda_{h}^{k-1} \lambda_{v}^{j}
$$

we find

$$
\lambda_{h}\left(\sum_{i=0}^{\infty} \sum_{j=0}^{\infty} x_{i+1, j} \lambda_{h}^{i} \lambda_{v}^{j}\right)=X\left(\lambda_{h}, \lambda_{v}\right)-\sum_{j=0}^{\infty} x_{0, j} \lambda_{v}^{j} .
$$

Similarly

$$
\begin{aligned}
\lambda_{v}\left(\sum_{i=0}^{\infty} \sum_{j=0}^{\infty} x_{i, j+1} \lambda_{h}^{i} \lambda_{v}^{j}\right)= & X\left(\lambda_{h}, \lambda_{v}\right)-\sum_{i=0}^{\infty} x_{i, 0} \lambda_{h}^{i} \\
\lambda_{h} \lambda_{v}\left(\sum_{i=0}^{\infty} \sum_{j=0}^{\infty} x_{i+1, j+1} \lambda_{h}^{i} \lambda_{v}^{j}\right)= & X\left(\lambda_{h}, \lambda_{v}\right)-\sum_{j=0}^{\infty} x_{0, j} \lambda_{v}^{j} \\
& -\sum_{i=0}^{\infty} x_{i, 0} \lambda_{h}^{i}+x_{0,0}
\end{aligned}
$$

(see also [16]). By writing the model (6) in terms of power series, we therefore get

$$
\begin{aligned}
& H\left(\lambda_{h}, \lambda_{v}\right) X\left(\lambda_{h}, \lambda_{v}\right) \\
& =V\left(\lambda_{h}, \lambda_{v}\right) U\left(\lambda_{h}, \lambda_{v}\right)+\left(E-A_{1} \lambda_{v}\right) \sum_{j=0}^{\infty} x_{0, j} \lambda_{v}^{j} \\
& \quad+\left(E-A_{2} \lambda_{h}\right) \sum_{i=0}^{\infty} x_{i, 0} \lambda_{h}^{i}-E x_{0,0} \\
& \quad-B_{1} \lambda_{v} \sum_{j=0}^{\infty} u_{0, j} \lambda_{v}^{j}-B_{2} \lambda_{h} \sum_{i=0}^{\infty} u_{i, 0} \lambda_{h}^{i} .
\end{aligned}
$$

On the other hand, if $x_{i,-1}=x_{-1, j}=0$ and $u_{i,-1}=u_{-1, j}=0$ for $i, j \in \mathbb{N}$, it follows by (6) that

$$
\begin{aligned}
& B_{2} u_{i-1,0}=E x_{i, 0}-A_{2} x_{i-1,0}, \quad \text { for } i=1,2, \ldots \\
& B_{1} u_{0, j-1}=E x_{0, j}-A_{1} x_{0, j-1}, \quad \text { for } j=1,2, \ldots \\
& x_{0,0}=0
\end{aligned}
$$


and consequently

$$
\begin{aligned}
B_{1} \lambda_{v} & \sum_{j=0}^{\infty} u_{0, j} \lambda_{v}^{j}+B_{2} \lambda_{h} \sum_{i=0}^{\infty} u_{i, 0} \lambda_{h}^{i} \\
= & \sum_{j=1}^{\infty}\left(E x_{0, j}-A_{1} x_{0, j-1}\right) \lambda_{v}^{j}+\sum_{i=1}^{\infty}\left(E x_{i, 0}-A_{2} x_{i-1,0}\right) \lambda_{h}^{i} \\
= & \left(E-A_{1} \lambda_{v}\right) \sum_{j=0}^{\infty} x_{0, j} \lambda_{v}^{j}+\left(E-A_{2} \lambda_{h}\right) \sum_{i=0}^{\infty} x_{i, 0} \lambda_{h}^{i} \\
& -2 E x_{0,0} .
\end{aligned}
$$

Therefore, (6) expressed in terms of formal power series becomes

$$
\begin{aligned}
H\left(\lambda_{h}, \lambda_{v}\right) X\left(\lambda_{h}, \lambda_{v}\right) & =V\left(\lambda_{h}, \lambda_{v}\right) U\left(\lambda_{h}, \lambda_{v}\right) \\
Y\left(\lambda_{h}, \lambda_{v}\right) & =C X\left(\lambda_{h}, \lambda_{v}\right)+D U\left(\lambda_{h}, \lambda_{v}\right)
\end{aligned}
$$

leading to

$\left(\operatorname{det} H\left(\lambda_{h}, \lambda_{v}\right)\right) Y\left(\lambda_{h}, \lambda_{v}\right)$

$=\left(C \operatorname{adj} H\left(\lambda_{h}, \lambda_{v}\right) V\left(\lambda_{h}, \lambda_{v}\right)+D \operatorname{det} H\left(\lambda_{h}, \lambda_{v}\right)\right) U\left(\lambda_{h}, \lambda_{v}\right)$

which is clearly equivalent to (5) in light of (11) and (12), modulo common terms in the form $\lambda_{h}^{n(m-n)}$ for $n<m$ and $\lambda_{v}^{m(n-m)}$ for $n>m$.

By substituting the complex variables $z_{1}$ and $z_{2}$ for the indeterminates $\lambda_{h}$ and $\lambda_{v}$ used in the definition of the power series representation of a 2-D signal, we recall that a necessary and sufficient condition for the latent variable model (6) to be asymptotically stable for any set of admissible boundary conditions is that the determinant of $H\left(z_{1}, z_{2}\right)$ differs from zero for all $0<\left|z_{1}\right| \leq 1$ and $0<\left|z_{2}\right| \leq 1$ (see [35, Th. 3]). Since, in Section III, it is proved that every set of boundary conditions (7) is admissible for the latent variable realization in Theorem 2.1 , the condition $\operatorname{det} H\left(z_{1}, z_{2}\right) \neq 0$ for all $0<\left|z_{1}\right| \leq 1$ and $0<\left|z_{2}\right| \leq 1$ guarantees asymptotic stability of (6) for any boundary condition (7).

Consider the input-output process (5). Assume that the polynomial $p\left(z_{1}, z_{2}\right)$ has no nonessential singularities of the second kind on the unit bitorus $\mathbb{T}:=\left\{\left(z_{1}, z_{2}\right) \in \mathbb{C} \times \mathbb{C}|| z_{1}|=1,| z_{2} \mid=\right.$ $1\} .{ }^{1}$ Then, consider that the input-output process (5) is BIBO stable if and only if $p\left(z_{1}, z_{2}\right)$ differs from zero for all $\left|z_{1}\right| \leq 1$ and $\left|z_{2}\right| \leq 1$, [3], [9]. Now, suppose that (5) is BIBO stable. In this case, the corresponding latent variable model given in Theorem 2.1 is asymptotically stable. To see this, let, for example, $n<m$. In view of the proof of Theorem 2.1, we find that det $H\left(z_{1}, z_{2}\right)=z_{1}^{n(m-n)} p\left(z_{1}, z_{2}\right)$. For all $0<\left|z_{1}\right| \leq 1$, the complex number $z_{1}^{n(m-n)}$ differs from zero, while $p\left(z_{1}, z_{2}\right)$ differs from zero for all $\left|z_{1}\right| \leq 1$ and $\left|z_{2}\right| \leq 1$. As a result

$z_{1}^{n(m-n)} p\left(z_{1}, z_{2}\right) \neq 0, \quad$ for $0<\left|z_{1}\right| \leq 1$ and $0<\left|z_{2}\right| \leq 1$

which guarantees asymptotic stability of the latent variable model given in Theorem 2.1 in view of [35, Th. 3].

Example 2: Consider a simple case where $(n, m)=(2,3)$. By Theorem 2.1, we have (15), shown at the bottom of the page, and

$$
V\left(\lambda_{h}, \lambda_{v}\right)=\left[\begin{array}{c}
\bar{b}_{2,3} \lambda_{h} \lambda_{v} \\
\bar{b}_{1,3} \lambda_{h} \lambda_{v}+\bar{b}_{0,3} \lambda_{v} \\
\bar{b}_{2,2} \lambda_{h} \lambda_{v} \\
\bar{b}_{2,1} \lambda_{h} \lambda_{v}+\bar{b}_{2,0} \lambda_{h} \\
\bar{b}_{1,2} \lambda_{h} \lambda_{v}+\bar{b}_{0,2} \lambda_{v} \\
\bar{b}_{1,1} \lambda_{h} \lambda_{v}+\bar{b}_{1,0} \lambda_{h}+\bar{b}_{0,1} \lambda_{v}
\end{array}\right]
$$

A simple computation shows that

$$
\begin{aligned}
\operatorname{det} H\left(\lambda_{h}, \lambda_{v}\right) & =\lambda_{h}^{2} \sum_{i=0}^{2} \sum_{j=0}^{3} a_{i, j} \lambda_{h}^{i} \lambda_{v}^{j} \\
C \operatorname{adj} H\left(\lambda_{h}, \lambda_{v}\right) & =\lambda_{h}^{2}\left[\begin{array}{llllll}
\lambda_{h} \lambda_{v}^{2} & \lambda_{v}^{2} & \lambda_{h} \lambda_{v} & \lambda_{h} & \lambda_{v} & 1
\end{array}\right] .
\end{aligned}
$$

${ }^{1}$ When $p\left(z_{1}, z_{2}\right)$ has nonessential singularities of the second kind on the unit bitorus, the input-output process may be bounded-input-bounded-output (BIBO) stable, but any Fornasini-Marchesini realization will result to nonasymptotic stability [2]. A well-known example of such a process is the one described by the transfer function $G\left(z_{1}, z_{2}\right)=\left(\left(1-z_{1}\right)^{8}(1-\right.$ $\left.\left.z_{2}\right)^{8}\right) /\left(2-z_{1}-z_{2}\right)$, which is BIBO stable, even though it has a nonessential singularity of the second kind for $z_{1}=z_{2}=1$. Any latent variable model realizing $G\left(z_{1}, z_{2}\right)$, however, is such that the determinant of $H\left(z_{1}, z_{2}\right)=E-A_{0} z_{1} z_{2}-A_{1} z_{2}-A_{2} z_{1}$ contains the polynomial $2-z_{1}-z_{2}$ as a factor, so the condition for asymptotic stability of the realization is never satisfied. This is true also for implicit Fornasini-Marchesini models, as shown explicitly in [35, Lemma 3].

$$
H\left(\lambda_{h}, \lambda_{v}\right)=\left[\begin{array}{cccccc}
\lambda_{h} & 0 & 0 & 0 & 0 & a_{2,3} \lambda_{h} \lambda_{v} \\
0 & \lambda_{h} & 0 & 0 & 0 & a_{1,3} \lambda_{h} \lambda_{v}+a_{0,3} \lambda_{v} \\
-\lambda_{h} \lambda_{v} & 0 & 1 & 0 & 0 & a_{2,2} \lambda_{h} \lambda_{v} \\
0 & 0 & 0 & 1 & 0 & a_{2,1} \lambda_{h} \lambda_{v}+a_{2,0} \lambda_{h} \\
0 & -\lambda_{h} \lambda_{v} & 0 & 0 & 1 & a_{1,2} \lambda_{h} \lambda_{v}+a_{0,2} \lambda_{v} \\
0 & 0 & -\lambda_{h} \lambda_{v} & -\lambda_{h} & -\lambda_{v} & 1+a_{1,1} \lambda_{h} \lambda_{v}+a_{0,1} \lambda_{v}+a_{1,0} \lambda_{h}
\end{array}\right]
$$


Therefore, it is readily seen that

$$
C \operatorname{adj} H\left(\lambda_{h}, \lambda_{v}\right) V\left(\lambda_{h}, \lambda_{v}\right)=\lambda_{h}^{2} \sum_{i=0}^{2} \sum_{j=0}^{2} \bar{b}_{i, j} \lambda_{h}^{i} \lambda_{v}^{j} .
$$

Thus, (15) is a realization of (5) with $n=2$ and $m=3$.

\section{RECURSIBILITY}

In this section, we show that, with boundary conditions (7), when the structure of the matrices appearing in (6) is as given in Theorem 2.1, the solution of (6) is unique and can be determined recursively.

For the $n=m$ case, the matrix $E$ in the realization (6) is the identity matrix. The model thus obtained is therefore a Fornasini-Marchesini model in Kurek form [20]. As such, the latent variable $x$ can be computed recursively when the boundary conditions (7) are imposed.

For the $n<m$ case, the matrices appearing in the realization (6) are in the form given in (8). We may then comformably partition the latent variable $x$ to obtain

$$
\begin{aligned}
x_{i, j+1}^{\prime}= & A_{0}^{22} x_{i, j}^{\prime}+A_{0}^{21} x_{i, j}^{\prime \prime}+A_{1}^{21} x_{i+1, j}^{\prime \prime} \\
& +B_{0}^{2} u_{i, j}+B_{1}^{2} u_{i+1, j} \\
x_{i+1, j+1}^{\prime \prime}= & A_{0}^{12} x_{i, j}^{\prime}+A_{0}^{11} x_{i, j}^{\prime \prime}+A_{1}^{11} x_{i+1, j}^{\prime \prime}+A_{2}^{11} x_{i, j+1}^{\prime \prime} \\
& +B_{0}^{1} u_{i, j}+B_{1}^{1} u_{i+1, j}+B_{2}^{1} u_{i, j+1} \\
y_{i, j}= & x_{i, j}^{\prime \prime} .
\end{aligned}
$$

Consider for simplicity that (17)-(19) are defined over the bounded frame $[-1, N] \times[-1, M]$. Let the boundary conditions associated with (17) and (18) be as in (7), so that, in particular, $x^{\prime}$ is zero on $[-1, N] \times\{-1\}$, while $x^{\prime \prime}$ and $u$ are zero on $[-1, N] \times\{-1\} \cup\{-1\} \times[-1, M]$. All this corresponds to fixing the value of $x^{\prime}$ on $\{-1\} \times[-1, \infty)$, but these values do not affect the solution of (17) and (18). Note that $x_{0,0}^{\prime}$ can be computed from (17), since $x_{0,-1}^{\prime}, x_{0,-1}^{\prime \prime}, x_{1,-1}^{\prime \prime}, u_{0,-1}$, and $u_{1,-1}$ are zero. It clearly follows that $x_{0,0}^{\prime}=0$. Similarly, since all the values of $x^{\prime}, x^{\prime \prime}$, and $u$ are zero on $[-1, N] \times\{-1\}$, it follows that, by (17), the component $x_{i, 0}^{\prime}$ can be computed (and its value is zero) for all $i=0,1, \ldots, N-1$. Notice that the value $x_{N, 0}^{\prime}$ does not influence the output over the bounded frame of interest due to the fact that $y$ depends on $x^{\prime}$ only through $x^{\prime \prime}$ in view of (19). We can now compute $x^{\prime \prime}$ on the same row, i.e., $j=0$. In fact, now, $x_{i, 0}^{\prime \prime}$ can be computed for all $i=0, \ldots, N$ by using (18), since $x_{i-1,-1}^{\prime}, x_{i-1,-1}^{\prime \prime}, x_{i,-1}^{\prime \prime}$, $x_{i-1,0}^{\prime \prime}, u_{i-1,-1}, u_{i,-1}$, and $u_{i-1,0}$ are all given. This yields $y_{i, 0}$ for all $i=0, \ldots, N$. At this point, one may proceed with the computation of $x_{i, 1}^{\prime}$ for all $i=0, \ldots, N-1$ by using (17) again. In fact, to that end, the values $x_{i, 0}^{\prime}, x_{i, 0}^{\prime \prime}, x_{i+1,0}^{\prime \prime}, u_{i, 0}$, and $u_{i+1,0}$ are required. These values have been computed in the previous steps. Then, again, by exploiting (18), one can compute $x_{i, 1}^{\prime \prime}$ (and then also $y_{i, 1}$ ) for all $i=0, \ldots, N$. This procedure for the computation of $x_{i, j}^{\prime}$ and $x_{i, j}^{\prime \prime}$ for all $i=0, \ldots, N-1$ and $i=0, \ldots, N$, respectively, can be repeated for all $j=1,2, \ldots, M$, so that the output can be recursively computed all over the bounded frame. Notice that, even if the latent variable component $x_{i, j+1}^{\prime}$ depends on the input $u_{i+1, j}$, this does not affect the possibility of recursively computing the output on the bounded frame of interest, given the input, since, as mentioned earlier, the output $y$ does not depend explicitly on $x^{\prime}$ although indirectly through $x^{\prime \prime}$.

For the $n>m$ case, the realization is in the form of (9). As previously observed, (9) becomes

$$
\begin{aligned}
x_{i+1, j}^{\prime}= & A_{0}^{22} x_{i, j}^{\prime}+A_{0}^{21} x_{i, j}^{\prime \prime}+A_{2}^{21} x_{i, j+1}^{\prime \prime} \\
& +B_{0}^{2} u_{i, j}+B_{1}^{2} u_{i, j+1} \\
x_{i+1, j+1}^{\prime \prime}= & A_{0}^{12} x_{i, j}^{\prime}+A_{0}^{11} x_{i, j}^{\prime \prime}+A_{1}^{11} x_{i+1, j}^{\prime \prime}+A_{2}^{11} x_{i, j+1}^{\prime \prime} \\
& +B_{0}^{1} u_{i, j}+B_{1}^{1} u_{i+1, j}+B_{2}^{1} u_{i, j+1} \\
y_{i, j}= & x_{i, j}^{\prime \prime}
\end{aligned}
$$

by which it can be shown that the values of $x^{\prime}$ and $x^{\prime \prime}$ (and, therefore, $y$ as well) can be recursively computed on the bounded frame $[0, N] \times[0, M]$, given the input. If the values of $x^{\prime}$ are zero on $\{-1\} \times[-1, M]$, as well as that of $x^{\prime \prime}$ and $u$ on $[-1, N] \times$ $\{-1\} \cup\{-1\} \times[-1, M]$, by using the first equation, we can compute $x^{\prime}$ on $\{0\} \times[-1, M]$, and then, the second equation may be used to compute $x^{\prime \prime}$ on $\{0\} \times[-1, M]$, and so on. That is, calculation proceeds column by column instead of row by row, as in the $n<m$ case.

It turns out that, in all three cases, when the boundary conditions of (6) are defined in a suitable way as in (7), the latent variable update equation of (6) can be iterated recursively in order to find $x_{i, j}$ for all $i, j \geq 0$.

\section{Realization Size Reduction}

Theorem 2.1 provides a latent variable realization of size $n \cdot m$ for a general 2-D process described by the input-output relation (5). This means that the latent variable $x$ is a vector of dimension $n \cdot m$. If some of the coefficients $a_{i, j}$ and $b_{i, j}$ in (5) are zero, the size of the realization may be reduced further by removing some components of the latent variable $x$. Denote the $k$ th element of $x$ by $x[k]$. If $x[k]$ is removed from $x$, then the corresponding $k$ th row of $A_{i}$ and $B_{i}$ and the $k$ th column of $A_{i}$ and $C(i=0,1,2)$ are deleted so that a realization with smaller size is obtained. The following algorithm proposes a method for the reduction of the size of the realization based on the coefficients of particular models.

Algorithm 1: Denote the $(k, l)$ th entry of matrix $H\left(\lambda_{h}, \lambda_{v}\right)$ by $H[k, l]$. Consider the following series of sets denoted by $\rho_{k}$, where $k=1,2,3, \ldots, n m-1$ :

$$
\begin{aligned}
\rho_{k}=\left\{A_{0}[k, n m], A_{1}[k, n m], A_{2}[k, n m], B_{0}[k, 1],\right. \\
\left.B_{1}[k, 1], B_{2}[k, 1]\right\} \cup \rho_{s_{1}} \cup \rho_{s_{2}} \cup \cdots \cup \rho_{s_{j}}
\end{aligned}
$$

if there exists $s_{i} \in\{1, \ldots, k-1\}, i=1,2, \ldots, j$ such that

$$
\left|A_{0}\left[k, s_{i}\right]\right|+\left|A_{1}\left[k, s_{i}\right]\right|+\left|A_{2}\left[k, s_{i}\right]\right| \neq 0 .
$$

If there does not exist $s_{i}$ such that (21) holds, then

$$
\begin{array}{r}
\rho_{k}=\left\{A_{0}[k, n m], A_{1}[k, n m], A_{2}[k, n m],\right. \\
\left.B_{0}[k, 1], B_{1}[k, 1], B_{2}[k, 1]\right\} .
\end{array}
$$

For a certain $k$, the $k$ th element of $x$ can be deleted if $\rho_{k}=$ $\{0\}$.

To show the basic idea of this algorithm, consider the realization (15) for the $(n, m)=(2,3)$ case. It can be found that, for 
the special case where $a_{2,3}=0$ and $\bar{b}_{2,3}=0, \rho_{1}=\{0\}$ because the $(1,6)$ th entries of all matrices are zero, and there does not exist $s_{i} \in\{0, \ldots, k-1\}$ such that (21) is satisfied because $k$ is just 1. By Algorithm 1, the first row and the first column of $H\left(\lambda_{h}, \lambda_{v}\right)$ can be removed, and the new $H\left(\lambda_{h}, \lambda_{v}\right)$ is denoted by $\hat{H}\left(\lambda_{h}, \lambda_{v}\right)$. Then

$$
\begin{aligned}
\operatorname{det}\left(\hat{H}\left(\lambda_{h}, \lambda_{v}\right)\right) & =\lambda_{h} \sum_{i=0}^{2} \sum_{j=0}^{3} a_{i, j} \lambda_{h}^{i} \lambda_{v}^{j} \\
{\left[\begin{array}{lllll}
0 & \cdots & 0 & 1
\end{array}\right] \operatorname{adj}\left(\hat{H}\left(\lambda_{h}, \lambda_{v}\right)\right) } & =\lambda_{h}\left[\begin{array}{lllll}
\lambda_{v}^{2} & \lambda_{h} \lambda_{v} & \lambda_{h} & \lambda_{v} & 1
\end{array}\right] .
\end{aligned}
$$

Thus, the corresponding reduced size realization still represents (5). Consider another special case where $a_{1,3}, a_{0,3}, \bar{b}_{13}$, and $\bar{b}_{0,3}$ are all equal to zero. Also, in this case, the deletion of the corresponding rows and columns from $H\left(\lambda_{h}, \lambda_{v}\right)$ does not change the input-output relationship.

However, when the coefficients $a_{2,2}$ and $\bar{b}_{22}$ are zero, the third row and the third column of $H\left(\lambda_{h}, \lambda_{v}\right)$ cannot be removed in general because the entry $H[3,1]$ may not be zero. Such deletion, which leads to a new $H\left(\lambda_{h}, \lambda_{v}\right)$ denoted by $\tilde{H}\left(\lambda_{h}, \lambda_{v}\right)$, would lead to

$$
\begin{aligned}
& \operatorname{det}\left(\tilde{H}\left(\lambda_{h}, \lambda_{v}\right)\right) \\
& =\lambda_{h}^{2}\left(\sum_{i=0}^{1} \sum_{j=0}^{3} a_{i, j} \lambda_{h}^{i} \lambda_{v}^{j}+a_{2,0} \lambda_{h}^{2}+a_{2,1} \lambda_{h}^{2} \lambda_{v}\right) .
\end{aligned}
$$

Obviously, the term $a_{2,3} \lambda_{h}^{2} \lambda_{v}^{3}$ also disappears from $\operatorname{det}\left(\tilde{H}\left(\lambda_{h}, \lambda_{v}\right)\right)$ with the term $a_{2,2} \lambda_{h}^{2} \lambda_{v}^{2}$, and $\bar{b}_{2,3} \lambda_{h}^{2} \lambda_{v}^{3}$ would not appear, since

$$
\left[\begin{array}{llll}
0 & \cdots & 0 & 1
\end{array}\right] \operatorname{adj}\left(\tilde{H}\left(\lambda_{h}, \lambda_{v}\right)\right)=\lambda_{h}^{2}\left[\begin{array}{lllll}
0 & \lambda_{v}^{2} & \lambda_{h} & \lambda_{v} & 1
\end{array}\right] .
$$

Therefore, whether the third row and the third column can be deleted depends on not only $a_{2,2}$ and $\bar{b}_{22}$ but also $a_{2,3}$ and $\bar{b}_{2,3}$. Consistently, in Algorithm 1 , the set $\rho_{3}$ is defined as $\left\{a_{2,2}, \bar{b}_{22}\right\} \cup \rho_{1}=\left\{a_{2,2}, \bar{b}_{22}, a_{2,3}, \bar{b}_{2,3}\right\}$, and all the coefficients in $\rho_{3}$ must be zero to allow the deletion of the third component of the latent variable $x$.

Stated differently, for a particular $k$, even if $H[k, n m]=0$ and, correspondingly, $V[k]=0$, we need to check if $H[k, s]=$ 0 for $s=1, \ldots, k-1$. Notice that we do not care about $H[k, s]$ for $s=k+1, \ldots, n m-1$, because these values are all zeros according to Theorem 2.1.

To further verify and illustrate the proposed algorithm, consider the following example.

Example 3: Consider a 2-D system ruled by

$$
\begin{aligned}
& \left(1+a_{0,1} \lambda_{v}+a_{1,0} \lambda_{h}+a_{1,1} \lambda_{h} \lambda_{v}+a_{2,4} \lambda_{h}^{2} \lambda_{v}^{4}\right) Y\left(\lambda_{h}, \lambda_{v}\right) \\
& \quad=\left(b_{0,1} \lambda_{v}+b_{1,0} \lambda_{h}+b_{1,1} \lambda_{h} \lambda_{v}+b_{2,4} \lambda_{h}^{2} \lambda_{v}^{4}\right) U\left(\lambda_{h}, \lambda_{v}\right) .
\end{aligned}
$$

By Theorem 2.1, for the general $(2,4)$ th-order system, a realization in the form of (6) of order 8 can be obtained with the equation shown at the bottom of the page. According to Algorithm 1 , we have

$$
\begin{aligned}
& \rho_{1}=\left\{a_{24}, b_{24}\right\} \\
& \rho_{2}=\left\{a_{04}, a_{14}, b_{04}, b_{14}\right\} \\
& \rho_{3}=\left\{a_{23}, b_{23}\right\} \cup \rho_{1} \\
& \rho_{4}=\left\{a_{03}, a_{13}, b_{03}, b_{13}\right\} \cup \rho_{2} \\
& \rho_{5}=\left\{a_{22}, b_{22}\right\} \cup \rho_{3} \\
& \rho_{6}=\left\{a_{20}, a_{21}, b_{20}, b_{21}\right\} \\
& \rho_{7}=\left\{a_{02}, a_{12}, b_{01}, b_{12}\right\} \cup \rho_{4} .
\end{aligned}
$$

For the particular system (23), it is found that all elements in $\rho_{2}, \rho_{4}, \rho_{6}$, and $\rho_{7}$ are zero. As such, the second, fourth, sixth, and seventh components of the latent variable $x$ can be removed. As a result of this, the realization becomes

$$
E=\left[\begin{array}{cccc}
0 & 0 & 0 & 0 \\
0 & 0 & 0 & 0 \\
0 & 0 & 1 & 0 \\
0 & 0 & 0 & 1
\end{array}\right] \quad A_{0}=\left[\begin{array}{cccc}
0 & 0 & 0 & -a_{2,4} \\
1 & 0 & 0 & 0 \\
0 & 1 & 0 & 0 \\
0 & 0 & 1 & -a_{1,1}
\end{array}\right]
$$

$$
\begin{aligned}
& H\left(\lambda_{h}, \lambda_{v}\right)= {\left[\begin{array}{cccccccc}
\lambda_{h} & 0 & 0 & 0 & 0 & 0 & 0 & a_{2,4} \lambda_{h} \lambda_{v} \\
0 & \lambda_{h} & 0 & 0 & 0 & 0 & 0 & a_{1,4} \lambda_{h} \lambda_{v}+a_{0,4} \lambda_{v} \\
-\lambda_{h} \lambda_{v} & 0 & \lambda_{h} & 0 & 0 & 0 & 0 & a_{2,3} \lambda_{h} \lambda_{v} \\
0 & -\lambda_{h} \lambda_{v} & 0 & \lambda_{h} & 0 & 0 & 0 & a_{1,3} \lambda_{h} \lambda_{v}+a_{0,3} \lambda_{v} \\
0 & 0 & -\lambda_{h} \lambda_{v} & 0 & 1 & 0 & 0 & a_{2,2} \lambda_{h} \lambda_{v} \\
0 & 0 & 0 & 0 & 0 & 1 & 0 & a_{2,1} \lambda_{h} \lambda_{v}+a_{2,0} \lambda_{h} \\
0 & 0 & 0 & -\lambda_{h} \lambda_{v} & 0 & 0 & 1 & a_{1,2} \lambda_{h} \lambda_{v}+a_{0,2} \lambda_{v} \\
0 & 0 & 0 & 0 & -\lambda_{h} \lambda_{v} & -\lambda_{h} & -\lambda_{v} & 1+a_{1,1} \lambda_{h} \lambda_{v}+a_{0,1} \lambda_{v}+a_{1,0} \lambda_{h}
\end{array}\right] } \\
& V\left(\lambda_{h}, \lambda_{v}\right)= \\
&\left.\begin{array}{c}
\bar{b}_{2,4} \lambda_{h} \lambda_{v} \\
\bar{b}_{1,4} \lambda_{h} \lambda_{v}+\bar{b}_{0,4} \lambda_{v} \\
\bar{b}_{2,3} \lambda_{h} \lambda_{v} \\
\bar{b}_{1,3} \lambda_{h} \lambda_{v}+\bar{b}_{0,3} \lambda_{v} \\
\bar{b}_{2,2} \lambda_{h} \lambda_{v} \\
\bar{b}_{2,1} \lambda_{h} \lambda_{v}+\bar{b}_{2,0} \lambda_{h} \\
\bar{b}_{1,2} \lambda_{h} \lambda_{v}+\bar{b}_{0,2} \lambda_{v} \\
\bar{b}_{1,1} \lambda_{h} \lambda_{v}+\bar{b}_{1,0} \lambda_{h}+\bar{b}_{0,1} \lambda_{v}
\end{array}\right]
\end{aligned}
$$




$$
\begin{aligned}
A_{1} & =\left[\begin{array}{cccc}
0 & 0 & 0 & 0 \\
0 & 0 & 0 & 0 \\
0 & 0 & 0 & 0 \\
0 & 0 & 0 & -a_{0,1}
\end{array}\right] \quad A_{2}=\left[\begin{array}{cccc}
-1 & 0 & 0 & 0 \\
0 & -1 & 0 & 0 \\
0 & 0 & 0 & 0 \\
0 & 0 & 0 & -a_{1,0}
\end{array}\right] \\
B_{0} & =\left[\begin{array}{c}
b_{2,4} \\
0 \\
0 \\
b_{1,1}
\end{array}\right] \quad B_{1}=\left[\begin{array}{c}
0 \\
0 \\
0 \\
b_{0,1}
\end{array}\right] \quad B_{2}=\left[\begin{array}{c}
0 \\
0 \\
0 \\
b_{1,0}
\end{array}\right] \\
C & =\left[\begin{array}{llll}
0 & 0 & 0 & 1
\end{array}\right] .
\end{aligned}
$$

Remark 4.1: The algorithm described in this section can be applied for the order reduction of other latent variable models. For example, for FM-II models, we have that the sets $\rho_{k}$ for $k=1,2,3, \ldots, n m-1$ are defined as

$$
\begin{aligned}
\rho_{k}=\left\{A_{1}[k, n m], A_{2}[k, n m], B_{1}[k, 1],\right. & \left.B_{2}[k, 1]\right\} \\
& \cup \rho_{s_{1}} \cup \rho_{s_{2}} \cup \cdots \cup \rho_{s_{j}}
\end{aligned}
$$

where $s_{i} \in\{1, \ldots, k-1\}, i=1,2, \ldots, j$ satisfies $\left|A_{1}\left[k, s_{i}\right]\right|+$ $\left|A_{2}\left[k, s_{i}\right]\right| \neq 0$. If there does not exist $s_{i}$ such that $\left|A_{1}\left[k, s_{i}\right]\right|+$ $\left|A_{2}\left[k, s_{i}\right]\right| \neq 0$ holds, then

$$
\rho_{k}=\left\{A_{1}[k, n m], A_{2}[k, n m], B_{1}[k, 1], B_{2}[k, 1]\right\} .
$$

For a certain $k$, the $k$ th element of $x$ can be deleted if $\rho_{k}=$ $\{0\}$.

\section{CONCLUSION}

This paper investigates the possibility of representing a causal 2-D linear input-output process by means of an implicit latent variable model. The advantage of the proposed model is that it provides system matrices of much smaller size with respect to other realizations available in the literature. Indeed, for the general $(n, m)$ th-order 2-D system in the form of (5), the size of the realization by using the proposed model is $n \cdot m$. Importantly, this goal is achieved while maintaining recursibility of the latent variable model involved. For the Fornasini-Marchesini second model, on the other hand, the size of the realization given in [33] is $n m+m+n$, which is the smallest dimension of all the realizations available in the literature using Fornasini-Marchesini realizations [3], [7].

Another contribution of this paper is an algorithm to further reduce the size of the realization when some of the coefficients of the transfer function are zero. Furthermore, the idea of this algorithm can also be applied to the model reduction of the traditional Fornasini-Marchesini realizations.

Note that, although this paper is focused on the realization of 2-D SISO systems, following from the traditional realization of 1-D multi-input-multi-output (MIMO) systems [19, Ch. 6.1], the realization given in Theorem 2.1 can be easily extended to the realization of 2-D multi-input-single-output (MISO) or MIMO systems.

For the MISO case, the realization given in Theorem 2.1 does not change, except that the dimension of the realization matrices $B_{k}(k=0,1,2)$ becomes $n m \times \kappa_{\text {input }}$ instead of $n m \times 1$, where $\kappa_{\text {input }}$ is the number of inputs, since all parameters $b_{i, j}$ in (5) are no longer scalars but row vectors of dimension $1 \times \kappa_{\text {input }}$. This difference does not affect the recursibility of the realization, and the size reduction algorithm is applicable as well. Based on this, a MIMO system can be regarded as several MISO systems, and then, the matrices $A_{k}$ and $C_{k}(k=0,1,2)$ become block diagonal matrices, with each block pair corresponding to the relationship from the inputs to a certain output.

\section{REFERENCES}

[1] G. E. Antoniou, P. N. Paraskevopoulos, and S. J. Varoufakis, "Minimal state-space realization of factorable 2-D transfer functions," IEEE Trans. Circuits Syst., vol. 35, no. 8, pp. 1055-1058, Aug. 1988.

[2] M. Bisiacco, E. Fornasini, and G. Marchesini, "On some connections between BIBO and internal stability of two-dimensional filters," IEEE Trans. Circuits Syst., vol. 32, no. 9, pp. 948-953, Sep. 1985.

[3] M. Bisiacco, E. Fornasini, and G. Marchesini, "Dynamic regulation of 2-D systems: A state-space approach," Linear Algebra Appl., vol. 122-124, pp. 195-218, 1989.

[4] T. Bose, "Asymptotic stability of two-dimensional digital-filters under quantization," IEEE Trans. Signal Process., vol. 42, no. 5, pp. 1172-1177, May 1994

[5] C. W. Chen, J. S. H. Tsai, and L. S. Shieh, "Modeling of variable coefficient Roesser model for systems described by second-order partial differential equation," Circuits, Syst. Signal Process., vol. 22, no. 5, pp. 423-463, 2003.

[6] C. Du and L. Xie, $H_{\infty}$ Control and Filtering of Two-Dimensional Systems. Berlin, Germany: Springer-Verlag, 2002, vol. 278, Lecture Notes in Control \& Information Sciences.

[7] E. Fornasini and G. Marchesini, "State-space realization theory of twodimensional filters," IEEE Trans. Autom. Control, vol. AC-21, no. 4, pp. 484-492, Aug. 1976.

[8] E. Fornasini and G. Marchesini, "Doubly-indexed dynamical systems: State-space models and structural properties," Math. Syst. Theory, vol. 12 , no. 1 , pp. $59-72$, Dec. 1978.

[9] E. Fornasini and G. Marchesini, "Stability analysis of 2-D systems," IEEE Trans. Circuits Syst., vol. CAS-27, no. 12, pp. 1210-1217, Dec. 1980.

[10] K. Galkowski, "The Fornasini-Marchesini and the Roesser models: Algebraic methods for recasting," IEEE Trans. Autom. Control, vol. 41, no. 1, pp. 107-112, Jan. 1996.

[11] K. Galkowski, "A perspective on singularity in 2-D linear systems," Multidmensional Systems and Signal Processing, vol. 11, no. 1/2, pp. 83-108, Apr. 2000.

[12] K. Galkowski, State-Space Realizations of Linear 2-D Systems With Extensions to the General $n D(n>2)$ Case. New York: SpringerVerlag, 2001, Lecture Notes in Control and Information Sciences.

[13] T. Hinamoto, "2-D Lyapunov equation and filter design based on the Fornasini-Marchesini second model," IEEE Trans. Circuits Syst. I, Fundam. Theory Appl., vol. 40, no. 2, pp. 102-109, Feb. 1993.

[14] T. Kaczorek, Two-Dimensional Linear Systems. New York: SpringerVerlag, 1985.

[15] T. Kaczorek, "The singular general model of 2-D systems and its solution," IEEE Trans. Autom. Control, vol. 33, no. 11, pp. 1060-1061, Nov. 1988.

[16] T. Kaczorek, "General response formula and minimum energy control for the general singular model of 2-D systems," IEEE Trans. Autom. Control, vol. 35, no. 4, pp. 433-436, Apr. 1990.

[17] T. Kaczorek, Positive 1-D and 2-D Systems. New York: SpringerVerlag, 2002.

[18] T. Kaczorek, "State variable diagram method for determination of positive realizations of 2-D systems with delays," J. Autom., Mobile Robot. Intell. Syst., vol. 1, no. 2, pp. 6-12, 2007.

[19] T. Kailath, Linear Systems. Englewood Cliffs, NJ: Prentice-Hall, 1980.

[20] J. E. Kurek, "The general state-space model for a two-dimensional linear digital systems," IEEE Trans. Autom. Control, vol. AC-30, no. 6, pp. 600-602, Jun. 1985.

[21] K. S. Joo and T. Bose, "Two-dimensional periodically shift-variant dig ital filters," IEEE Trans. Circuits Syst. Video Technol., vol. 6, no. 1, pp. 97-107, Feb. 1996.

[22] B. C. Levy, M. B. Adams, and A. S. Willsky, "Solution and linear estimation of 2-D nearest-neighbor models," Proc. IEEE, vol. 78, no. 4, pp. 627-641, Apr. 1990.

[23] F. L. Lewis, "A review of 2-D implicit systems," Automatica, vol. 28, no. 2, pp. 345-354, Mar. 1992.

[24] F. L. Lewis and B. G. Mertzios, "On the analysis of two-dimensional discrete singular systems," Circuits Syst. Signal Process., vol. 11, no. 3, pp. 399-419, 1992. 
[25] C. Li and M. S. Fadali, "Optimal control of 2-D systems," IEEE Trans. Autom. Control, vol. 36, no. 2, pp. 223-228, Feb. 1991.

[26] W. S. Lu and E. B. Lee, "Stability analysis for two-dimensional systems via a Lyapunov approach," IEEE Trans. Circuits Syst., vol. 32, no. 1, pp. 61-68, Jan. 1985.

[27] D. Luenberger, "Dynamic equations in descriptor form," IEEE Trans. Autom. Control, vol. AC-22, no. 3, pp. 310-319, Jun. 1977.

[28] W. Marszalek, "Two-dimensional state-space discrete models for hyperbolic partial differential equations," Appl. Math. Model., vol. 8, no. 1, pp. 11-14, Feb. 1984.

[29] L. Ntogramatzidis, M. Cantoni, and R. Yang, "On the partial realisation of noncausal 2-D systems," IEEE Trans. Circuits Syst. I, Reg. Papers, vol. 54, no. 8, pp. 1800-1808, Aug. 2007.

[30] L. Ntogramatzidis, R. Yang, and M. Cantoni, On the Realisation of 2-D Linear Systems With Second-Order Fornasini-Marchesini Models Dept. Elect. Electron. Eng., Univ. Melbourne, Melbourne, Australia, 2005, Tech. Rep., TR06-001.

[31] R. Roesser, "A discrete state-space model for linear image processing," IEEE Trans. Autom. Control, vol. AC-20, no. 1, pp. 1-10, Feb. 1975.

[32] E. Rogers, K. Galkowski, and D. H. Owens, Control Systems Theory and Applications for Linear Repetitive Processes. Berlin, Germany: Springer-Verlag, 2001.

[33] L. Xu, L. Wu, Q. Wu, Z. Lin, and Y. Xiao, "On the realization of 2-D discrete systems by Fornasini-Marchesini model," Int. J. Control, Autom., Syst., vol. 3, no. 4, pp. 631-639, Dec. 2005.

[34] L. Xu, Q. Wu, Z. Lin, and Y. Xiao, "A new constructive procedure for 2-D coprime realization in Fornasini-Marchesini model," IEEE Trans. Circuits Syst. I, Reg. Papers, vol. 54, no. 9, pp. 2061-2069, Sep. 2007.

[35] Y. Zou and S. L. Campbell, "The jump behavior and stability analysis for 2-D singular systems," Multidimensional Syst. Signal Process., vol. 11, no. 4, pp. 321-338, Oct. 2000.

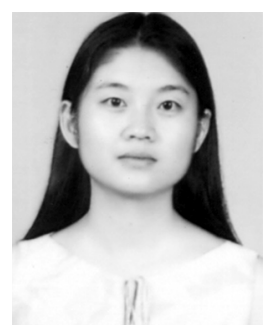

Ran Yang (M'05) received the B.E. degree in electrical engineering from Northeastern University, Shenyang, China, in 1996 and the M.Sc. and Ph.D. degrees in applied mathematics and electrical engineering from Shanghai Jiaotong University, Shanghai, China, in 1999 and 2001, respectively.

From 2001 to 2004, she was a Research Fellow with The University of Melbourne, Melbourne, Australia. Since 2005, she has been with the School of Information Science and Technology, Sun Yat-Sen University, Guangzhou, China, where she is currently an Associate Professor. Her research interests include multidimensional systems, descriptor systems, optimal estimation, and robust filtering and control.

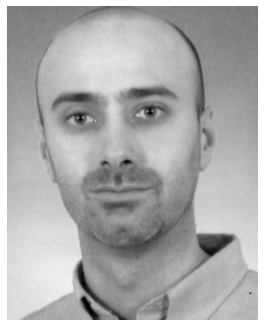

Lorenzo Ntogramatzidis received the "Laurea" degree (cum laude) in computer engineering and the $\mathrm{Ph} . \mathrm{D}$. degree in control and operations research from the University of Bologna, Bologna, Italy, in 2001 and 2005 , respectively.

From 2005 to 2008, he was with the Department of Electrical and Electronic Engineering, The University of Melbourne, Parkville, Australia. Since February 2009, he has been with the Department of Mathematics and Statistics, Curtin University of Technology, Perth, Australia, where he is currently an APD Research Fellow. His research interests are in the area of systems and control theory.

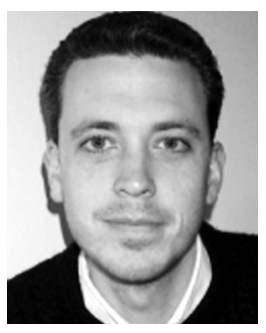

Michael Cantoni (S'95-A'98-M'00) received the B.E. (Hons. I) degree in electrical engineering and the B.Sc. degree in applied mathematics from The University of Western Australia, Perth, Australia, in 1995 and the Ph.D. degree in engineering from the University of Cambridge, Cambridge, U.K., in 1998.

From 1998 to 2000, he was a Research Associate with the Department of Engineering, University of Cambridge, and a Junior Research Fellow with St. John's College, Cambridge. Since 2000, he has been with the Department of Electrical and Electronic Engineering, The University of Melbourne, Melbourne, Australia, where he is currently an Associate Professor. His research interests include systems and control theory. 